\title{
Propuesta de optimización financiera en el proceso de tratamiento químico en torres de enfriamiento de la C.T. Francisco Pérez Ríos
}

\section{Proposal for financial optimization in the chemical treatment process in cooling towers of the C.T. Francisco Pérez Ríos}

PÉREZ-LUGO, Myrna Urith†๋*, SÁNCHEZ-TRUJILlO, Magda Gabriela, ACEVEDO-SÁNCHEZ, Ismael y CASTILLO-SERRANO, Erika

\author{
Universidad Tecnológica de Tula-Tepeji \\ Universidad Autónoma del Estado de Hidalgo
}

ID $1^{\text {er }}$ Autor: Myrna Urith, Pérez-Lugo / ORC ID: 0000-0002-4916-209X, CVU CONACYT ID: 689767

ID 1 ${ }^{\text {er }}$ Coautor: Magda Gabriela, Sánchez-Trujillo / ORC ID: 0000-0002-9093-1081, CVU CONACYT ID: 346119

ID $2^{\text {do }}$ Coautor: Ismael, Acevedo-Sánchez / ORC ID: 0000-0002-4167-3047, CVU CONACYT ID: 655755

ID $3^{\text {er }}$ Coautor: Erika, Castillo-Serrano / ORC ID: 0000-0001-8049-9902, CVU CONACYT ID: 689775

DOI: $10.35429 / J E D .2019 .21 .6 .14 .18$

Recibido: 10 de Junio, 2019; Aceptado 30 de Septiembre, 2019

\begin{abstract}
Resumen
El análisis técnico y financiero es esencial en todo proceso industrial para la toma de decisiones en la optimización de resultados. Sin embargo se requieren incrementar su eficiencia, así como un mayor aprovechamiento de recursos y observancia en materia de seguridad y confiabilidad en los sistemas de control de costos, estos son directrices que aumentan día con día. La producción de energía eléctrica tiene gran importancia en cuanto a progreso en México, debido a que tanto los negocios comerciales, las empresas industriales y hogares dependen en gran medida de su debida gestión. Este trabajo teórico es desarrollado a partir de una metodología técnicofinanciera del tratamiento químico del agua de la torre de enfriamiento, analizando su comportamiento financiero dentro del sistema eléctrico nacional de la Comisión Federal de Electricidad, en este caso se analiza la central termoeléctrica Francisco Pérez Ríos, que en la actualidad cuenta con cinco unidades generadoras de energía eléctrica, ubicada en Tula de Allende, Hidalgo, a partir de esta investigación se espera obtener ahorro en los costos del tratamiento químico en el agua de las torres de enfriamiento a partir de la información generada del área técnico-financiero.
\end{abstract}

\begin{abstract}
Technical and financial analysis are essential in every industrial process for decision-making in the optimization of results. However, they need to increase their efficiency, as well as greater use of security and reliability resources and enforcement in cost control systems, are guidelines that increase day by day, compared to this; electricity production is of great importance in terms of progress in Mexico, because both commercial businesses, industrial enterprises and households depend heavily on their proper management. This theoretical work is developed from a technical-financial methodology, carried out within the national electricity system of the Federal Electricity Commission, in this case analyzes the Francisco Pérez Ríos thermoelectric plant, which at present has five electric power generating units, located in Tula de Allende, Hidalgo, the contribution of this research is to seek the benefit in saving chemical treatment in the cooling towers from the information generated from the area Financial.
\end{abstract} enfriamiento de la C.T. Francisco Pérez Ríos. Revista de Desarrollo Económico. 2019. 6-21: 14-18

\footnotetext{
* Correspondencia del Autor (myrnaurith.perez@uttt.edu.mx)

$\dagger$ Investigador contribuyendo como primer autor.
} 


\section{Introducción}

En los grandes procesos químicos de la industria se generan enormes cantidades de calor que debe ser removido para que todas las unidades operen eficientemente. (Obregón Quiñones, Pertuz, \& Domínguez, 2017).

Obregón et al.(2017) menciona que los equipos utilizados en muchas empresas para disipar el calor son intercambiadores de calor y condensadores, debido a que se requiere tener procesos eficientes en cuanto al costo, razón por la cual gran cantidad del agua que se usa en muchos equipos de enfriamiento es recirculada y reutilizada.

En México se cuenta con la Comisión Federal de Electricidad (CFE), es una empresa paraestatal encargada de controlar, generar, transmitir y comercializar la energía eléctrica en todo el territorio mexicano (Ramos Gutiérrez \& Montenegro, 2012), con dicha empresa el gobierno federal maneja el parque eléctrico en el país.

Las empresas de la CFE son Centrales Eléctricas que a través de Redes Particulares son clasificadas en Empresas Productivas Subsidiarias (EPS) en Generación I, II, III, IV, V y VI (Comisión Federal de Eléctricidad, 2019).

Dentro de las EPS Generación I de la República Mexicana existe la Central Termoeléctrica (C.T.) Francisco Pérez Ríos, ubicada en el estado de Hidalgo en la región de Tula de Allende (CFE, 2017), la misma que será caso de estudio.

La generación de energía eléctrica dentro de la central termoeléctrica, dependen de la participación directa de equipos principales, tal es el caso del generador de vapor, turbina de vapor, generador eléctrico, transformadores principales, condensador, torre de enfriamiento y equipos auxiliares.

Vale la pena comentar nuevamente que en todo proceso químico industrial se aprovecha toda la materia y energía que se genera en él para economizar los procesos industriales (Comisión nacional para el uso eficiente de la energía, 2015). En el caso de la C.T., el agua es un recurso importante en el proceso de generación de energía eléctrica debido a que se le da diversos usos.

\section{Descripción del sistema}

Como se ha mencionado el proceso de generación de energía requiere de la participación de los equipos de torres de enfriamiento, cabe señalar que estos equipos forman parte del objeto de estudio para la presente investigación, las cuales juegan un papel importante porque el proceso se apoya de estos equipos para enfriar agua a grandes volúmenes y de esta forma extraer el calor que tiene el agua del proceso mediante la evaporación, este proceso es económico, lo que eleva su costo es el tratamiento químico que debe de tener el agua.

Se recurre al tratamiento químico del agua de torres de enfriamiento para mejorar y maximizar la eficiencia de los equipos en los cuales pasa el agua de enfriamiento y de esta forma reducir costos y energía, así como el consumo (suez, 2019).

El agua que se utiliza en las torres de enfriamiento cuenta con un suministro de pozos de la red no. 1 , de la red no. 2 y de su planta tratadora de aguas negras.

El agua contiene minerales como calcio, sílice, cloruros, fosfatos. Los minerales antes descritos en el agua de enfriamiento causan en los equipos que conforman el circuito de enfriamiento de la planta de proceso, problemas como corrosión, incrustación y ensuciamiento biológico.

Para el control de los problemas antes descritos en las torres de enfriamiento; es necesario se aplique un tratamiento químico integral con reactivos químicos, equipos de monitoreo, dosificación y control, para evitar que el proceso tenga afectaciones relacionadas con el agua de enfriamiento (Organización para el tratamiento de aguas, 2004).

Es importante el control de todos los parámetros del funcionamiento de la torre de enfriamiento, tiene la particularidad de consumir un volumen importante de agua debido a que se evapora, sufre arrastre y tiene purgas, el consumo aproximado por unidad es $1 \mathrm{l} / \mathrm{seg}$ MWH de tal manera que la C.T. requiere de 1500 $1 / \mathrm{s}$.

Esto es un dato importante, derivado de él depende el consumo del tratamiento químico. 
Se busca optimizar costos en el tratamiento químico del agua de las torres de enfriamiento, estas representan el segundo gasto fuerte que la empresa enfrenta, siendo el primero la compra del combustóleo como materia prima fundamental para lograr la generación de energía eléctrica por lo que la convierte en una producción costosa en este tipo de plantas convencionales.

Un modelo de optimización técnicofinanciero es una alternativa para mejorar la toma de decisiones en el proceso del tratamiento químico en torres de enfriamiento de la central termoeléctrica Francisco Pérez Ríos, en Tula de Allende, Hidalgo.

Aportando una propuesta para la generación de flujo de la empresa asegurando su sostenibilidad financiera.

\section{Características del proceso del agua de enfriamiento}

El agua negra tratada y el agua de pozos contienen sales minerales, estas se muestran en la siguiente tabla con un análisis típico:

\begin{tabular}{|l|r|r|l|}
\cline { 2 - 4 } \multicolumn{1}{c|}{} & \multicolumn{1}{c|}{$\begin{array}{c}\text { Agua negra } \\
\text { tratada }\end{array}$} \\
\hline $\mathrm{pH}$ & 7.5 & 7.5 & \\
\hline Conductividad & 1,650 & 1,400 & $\mu \mathrm{mohos} / \mathrm{cm}^{2}$ \\
\hline Alcalinidad F & 0 & 0 & $\mathrm{ppm}$ \\
\hline Alcalinidad M & 80 & 345 & $\mathrm{ppm}$ \\
\hline Dureza Total & 340 & 539 & $\mathrm{ppm}$ \\
\hline Dureza de Calcio & 110 & 345 & $\mathrm{ppm}$ \\
\hline Sílice & 65 & 66.5 & $\mathrm{ppm}$ \\
\hline Cloruros & 400 & 158 & $\mathrm{ppm}$ \\
\hline Fosfatos & 18 & 1.0 & $\mathrm{ppm}$ \\
\hline
\end{tabular}

Tabla 1 Sales minerales en el agua Fuente: $C F E$

El sistema de la torre de enfriamiento es un sistema cerrado, excepto por la reposición de agua perdida por evaporación, purga y arrastre, en donde el flujo teórico de reposición a considerar es de $636.5 \mathrm{~m}^{3} / \mathrm{hr}$.

Algunas de las particularidades de la torre de enfriamiento a considerar por el consumo importante de agua son:

\begin{tabular}{|l|r|}
\hline Volumen de agua de circulación & $3500 \mathrm{~m}^{3}$ \\
\cline { 2 - 2 } Flujo de agua de circulación & $21600 \mathrm{~m}^{3} / \mathrm{h}$ \\
\hline $\begin{array}{l}\text { Diferencial de temperatura } \\
\text { Evaporación }\end{array}$ & $12^{\circ} \mathrm{C}$ \\
\hline $\begin{array}{l}\text { Arrastre } \\
\text { Purga }\end{array}$ & $614.1 \mathrm{~m}^{3} / \mathrm{h}$ \\
\hline Repuesto & $64.8 \mathrm{~m}^{3} / \mathrm{h}$ \\
\hline
\end{tabular}

Tabla 1 Causas de perdida de agua en torre de enfriamiento

Fuente: $C F E$

\section{Ciclos de concentración en torres de enfriamiento}

Los ciclos de concentración están en función de las siguientes variables; como son la carga térmica a disipar de la planta de proceso (define la temperatura del agua de suministro y retorno), de la cantidad de minerales principalmente sílice que contiene el agua de repuesto, y del purgado de la torre de enfriamiento.

Durante la operación de la torre de enfriamiento se tiene evaporación y arrastre del agua, lo que causa que las sales minerales disueltas en el agua lleguen a concentrarse y precipitar formando incrustaciones, por lo tanto los ciclos de concentración es un indicativo de las impurezas presentes en el agua y determinan los requerimientos del purgado de la torre de enfriamiento.

El agua de repuesto a las torres de enfriamiento es inversamente proporcional a los ciclos de concentración; es decir a menores valores de ciclos de concentración se requiere mayor cantidad de agua de repuesto a las torres de enfriamiento y a valores mayores de ciclos de concentración se requiere menor cantidad de agua de repuesto.

$C=\frac{\text { concentración de sílice en el agua de recirculación }}{\text { concentración de sílice en el agua de repuesto }}$

\section{Metodología a desarrollar}

El control de tratamiento de agua a torres de enfriamiento es único. Las propiedades fisicoquímicas en el agua de las torres de enfriamiento varían mucho en todo el mundo. Las condiciones en las cuales es operada una torre de enfriamiento con respecto a temperatura, el tamaño, el caudal, las diversas condiciones del proceso influyen en la optimización financiera del tratamiento químico para su optimización de costos del mismo. 
Considerando todas las variables que se encuentran inmersas en la operación de la torre de enfriamiento se propone el siguiente modelo de optimización financiera para el tratamiento químico del agua en torres de enfriamiento.

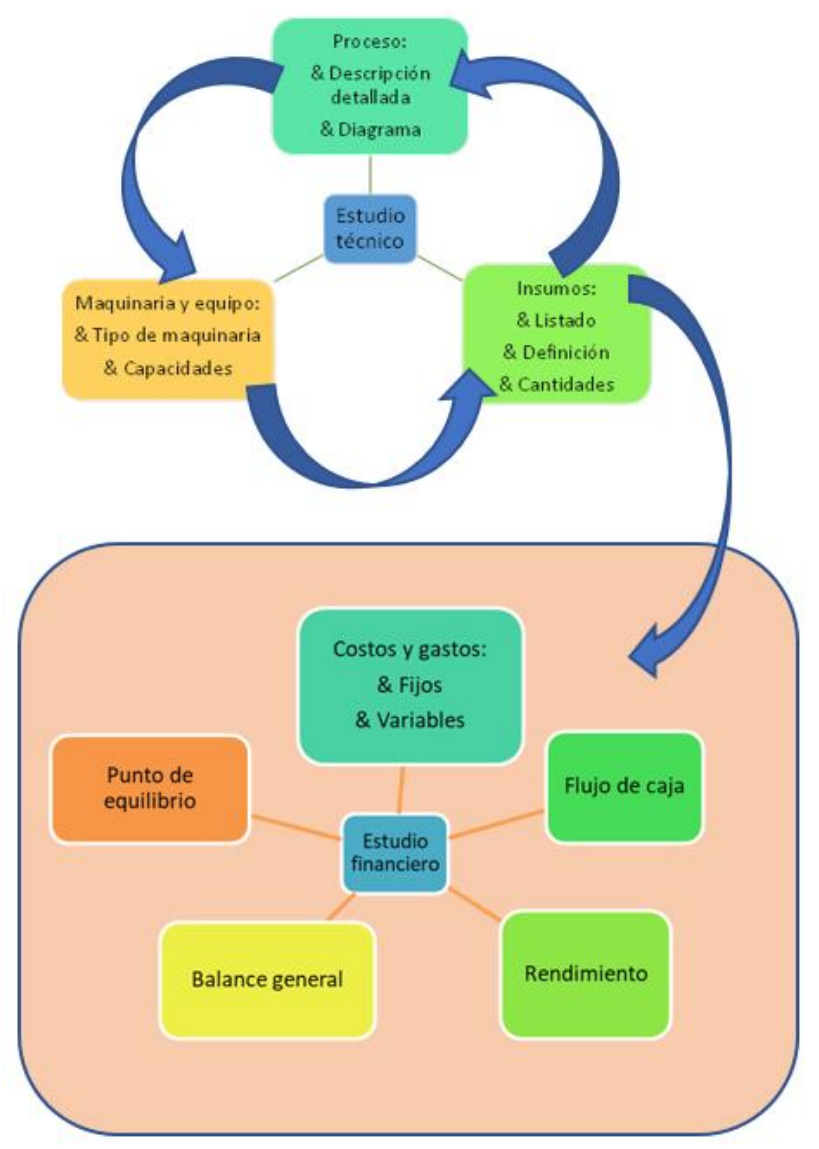

Figura 1 Metodología para estudio técnico y financiero Fuente: Elaboración Propia

\section{Resultados}

El cálculo del agua para la torre de enfriamiento es necesario para el control de los costos del agua y del tratamiento químico, considerando esto se espera determinar el balance de agua en las torres de enfriamiento con el flujo de agua de recirculación, evaporación y arrastre, purga y repuesto.

A partir del balance de agua en la torre de enfriamiento considerar los ciclos de concentración reales con los ciclos óptimos para obtener un comparativo de las variables optimas y reales y determinar costos. Más sin embargo no se debe de perder de vista las características fisicoquímicas del agua para la torre.

\section{Conclusiones}

Para proponer la optimización financiera se deben de desarrollar diferentes alternativas para el agua en base a los ciclos de concentración, debido a las pérdidas de agua que se presentan durante el proceso de enfriamiento; arrastre, purga y evaporación, entre menor sean los ciclos de concentración que se tengan mayor será el agua de repuesto que se necesitará para operar las torres. Los ciclos de concentración deben de estar controlados dentro de un rango determinado, encontrarse fuera de los ciclos establecidos generaría un mayor costo.

Para la aplicación del modelo de optimización técnico-financiera de costos en el tratamiento químico del agua de torre de enfriamiento es considerado hasta este momento una estimación de costos. Para el cálculo es indispensable contar con los precios por extracción de agua, el consumo eléctrico de las bombas y el precio de aplicación de tratamientos químicos.

\section{Referencias}

Comisión Federal de Eléctricidad. (26 de 08 de 2019). Gobiernos México. Recuperado el 26 de 08 de 2019, de Gobiernos México: https://www.gobiernosmexico.com.mx/gobiern o-federal/cfe-la-cfe-presenta-su-plan-denegocios-2017-2021/

Comisión nacional para el uso eficiente de la energía. (2015). Torres de enfriamiento. México.

Obregón Quiñones, L. G., Pertuz, J. C., \& Domínguez, R. A. (2017). Análisis del desempeño de una torre de enfriamiento a escala de laboratorio para diversos materiales de empaque, temperatura de entrada de agua y relación másica de flujo agua-aire . Prospect, 4252.

Organización para el tratamiento de aguas. (2004). Propuesta técnica. México.

Ramos Gutiérrez, L. d., \& Montenegro, F. M. (2012). La generación de energía eléctrica en México. Scielo Tecnología y ciencias del agua, 3(4). 
suez. (26 de 08 de 2019). Water Technologies \& Solutions. Recuperado el 26 de Agosto de 2019, de Water Technologies \& Solutions: https://www.suezwatertechnologies.mx/applicat ions/cooling-water-treatment 